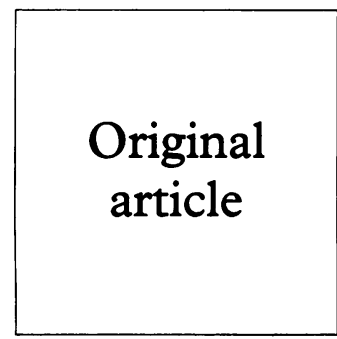

\title{
Evaluation of the microparticle enzyme immunoassay Abbott IMx Select Chlamydia and the importance of urethral site sampling to detect Chlamydia trachomatis in women
}

\author{
M K Brokenshire, P J Say, A H van Vonno, C Wong
}

Objective: To evaluate the commercial microparticle enzyme immunoassay (MEIA), Abbott IMx Select Chlamydia, for the detection of Chlamydia trachomatis in women and to compare its performance with endocervical cell culture. Also, to determine whether sampling the urethral site is an important part of chlamydial diagnosis in women.

Setting: The Auckland, Manukau, and Waitakere Sexual Health Clinics, Auckland, New Zealand and the Department of Clinical Microbiology, Auckland Hospital, Auckland, New Zealand.

Patients: The study population consisted of 622 consecutive women who attended the three sexual health clinics.

Methods: The IMx Chlamydia assay was performed on an IMx analyser, following a specimen treatment procedure. All reactive samples from the IMx Chlamydia assay were confirmed using the IMx Chlamydia blocking antibody reagent. The Syva direct fluorescent antibody (DFA) test was used to aid in resolving discrepancies. The cell culture technique was performed in shell vials using cycloheximide treated McCoy cells, which were stained using a fluorescein conjugated monoclonal antibody.

Results: When compared against the endocervical cell culture, the IMx Chlamydia had a sensitivity of $82 \cdot 1 \%(23 / 28)$ and a specificity of $99 \cdot 3 \%(590 / 594)$. When compared against an expanded gold standard, the IMx Chlamydia and endocervical cell culture had sensitivities of $84 \cdot 4 \%(27 / 32)$ and $87 \cdot 5 \%(28 / 32)$, specificities of $100 \%(590 / 590)$ and $100 \%(590 / 590)$, positive predictive values of $100 \%(27 / 27)$ and $100 \%(28 / 28)$, negative predictive values of $99 \cdot 2 \%$ (590/595) and 99.3\% (590/594), and accuracies of $99 \cdot 2 \%(617 / 622)$ and $99 \cdot 4 \%(618 / 622)$, respectively. The prevalence rate by endocervical cell culture and the expanded gold standard were $4.5 \%$ and $5 \cdot 1 \%$, respectively. Additional urethral cell culture testing revealed a further nine patients positive from this site only, giving a $28 \%$ (9/32) increase in the number of patients diagnosed for chlamydia, thus giving an overall prevalence of $6 \cdot 6 \%(41 / 622)$.

Conclusions: The IMx Chlamydia assay is an easy and rapid test to perform, it is cost effective, and shows similar performance to endocervical cell culture in the female population studied and is thus an excellent alternative to culture for the diagnosis of $C$ trachomatis. The study also showed the importance of urethral site sampling in these women, as endocervical testing alone will underestimate the prevalence of chlamydial genital infection.

(Genitourin Med 1997;73:498-502)

Keywords: Chlamydia trachomatis; microparticle; cell culture; urethra

\section{Introduction}

Chlamydia trachomatis is a leading cause of sexually transmitted disease around the world. The early diagnosis and treatment is important to prevent complications and to limit the spread of infection, as the sequelae may lead to endometritis, pelvic inflammatory disease, infertility, and ectopic pregnancy. The incidence of endocervical carriage in the Auckland sexual health clinic population has fallen dramatically from $24 \%$ in $1982^{1}$ to $5.3 \%$ in 1993 . The current low incidence and the relatively high asymptomatic rate in women raises concern about the provision of an optimum and cost effective test for the diagnosis of chlamydia infections.

Traditionally, the gold standard cell culture method was routinely used to identify the presence of chlamydia infections. The methodology involved with this is complex, requires considerable technical expertise viable organisms, and is time consuming, which means that it is not a suitable method for many laboratories to perform. Direct antigen tests, including the direct fluorescent antibody technique and the enzyme immunoassay, have largely replaced cell culture in the routine testing for $C$ trachomatis. ${ }^{2}$ There are now many non-culture antigen detection techniques available. ${ }^{3}$ Generally, these offer a rapid assay system which is easy to perform, cost effective, and accurate-important factors in an effective chlamydial screening programme. ${ }^{4}$ To maintain accuracy of the enzyme immunoassay tests, it is essential that confirmation tests are performed on reactives above the cut off and reactives in the grey zone region. The blocking antibody reagent test and/or direct fluorescent antibody technique, are available as confirmatory tests. 
The Abbott IMx Select Chlamydia (Abbott Laboratories, Abbott Park, IL, USA), a microparticle enzyme immunoassay (MEIA), was evaluated and compared with the cell culture for the detection of $C$ trachomatis in endocervical samples from patients routinely attending three sexual health clinics in the Auckland region.

The diagnosis of female chlamydia genital infection depends on the site sampled. The endocervical site has long been considered the primary site of infection, but there is now increasing awareness of the importance of the urethral site, in some patient populations, as sometimes being the only site from which chlamydia can be detected. Thus, multiple site sampling is an important consideration in the diagnosis of chlamydial infection in women.

\section{Materials and methods SUBJECTS}

The study population consisted of female patients attending three sexual health clinics of the Auckland region. These patients were from all strata of society, mostly self referrals, consisting of approximately $75 \%$ European, $22 \%$ Maori, $1 \%$ Polynesian, and $2 \%$ others. A total of 622 consecutive women, attending for the first time, participated in the study. During the routine history taking, the study was explained to the patient, who was also provided with information in writing and an informed consent form was signed before any specimens were taken. The study was performed during the months of September and October 1993, approval of which was granted by the North Health ethics committee.

\section{SPECIMEN COLLECTION}

Standard specimen collection techniques were used during the routine genital examination. Cotton tipped swab specimens were taken from the external os of the endocervix, for Gram staining of endocervical secretions and Neisseria gonorrhoeae direct culture. When clinically indicated, a Papanicolaou smear was taken using a modified Ayres spatula; in some cases an endocervical brush specimen was also taken.

Then, two chlamydia endocervical specimens were collected. Firstly, a Dacron tipped, wire shafted swab was inserted into the endocervical canal and rotated for 10 seconds to obtain a good scrape of columnar epithelial cells. The swab was then placed into a sucrose phosphate transport medium (Chlamydia Transwab, Medical Wire and Equipment Company, UK) and refrigerated at $4-8^{\circ} \mathrm{C}$ for a maximum of 24 hours. If processing was not performed within this time $-70^{\circ} \mathrm{C}$ storage was used for up to 5 days. Secondly, a Dacron tipped, wire shafted Abbott MEIA swab (supplied by the manufacturer) was used to collect another endocervical sample in an identical manner as above. The swab was then broken off into the Abbott MEIA transport tube, stored at $4-8^{\circ} \mathrm{C}$ and the immunoassay testing was performed within 7 days (according to manufacturer's instructions). After half of the female patients had been recruited into the study, the order of the endocervical swab taking was reversed, so that the MEIA swab was collected before the cell culture swab.

Also, swabs from the female urethra in the same study group were collected as is routine, but were stored and tested separately by the cell culture method. The purpose of this was to reaffirm the importance of multiple site sampling for chlamydia. Urethral sampling was also performed, initially with cotton tipped swabs for Gram stain of the urethral smear and direct culture for $N$ gonorrhoeae and then followed by a chlamydia transwab gently inserted $0.5 \mathrm{~cm}$ into the urethra and rotated with a scraping movement.

\section{CHLAMYDIA CELL CULTURE}

The method used was based on standard techniques for the isolation of $C$ trachomatis. ${ }^{56}$ Briefly, specimens were inoculated into shell vials containing McCoy cell monolayers. Following centrifugation, cycloheximide containing growth medium was added. A fluorescein conjugated monoclonal antibody (Pathfinder, Kallestad Diagnostics, MN, USA) was used to stain the monolayers after incubation and the presence of one or more characteristic apple green fluorescent inclusion bodies was considered positive.

\section{ABBOTT IMX CHLAMYDIA}

The Abbott IMx Select Chlamydia assay was performed using the IMx analyser according to the manufacturer's instructions. A detailed description and operation of this automated bench top analyser system has already been described. ${ }^{7}$ Briefly, specimens and controls underwent a pretreatment procedure by the addition of an extraction buffer and heat treatment at $100^{\circ} \mathrm{C}$ for 10 minutes. After cooling, the samples were filtered using a special Abbott STD filtration system and a $150 \mu \mathrm{l}$ aliquot of the filtrate was used in the assay. For the detection of chlamydia LPS, the IMx instrument automatically performed all the steps required for the analysis of the patient and control samples. A reactive specimen was defined as having a $\mathrm{S} / \mathrm{N}$ (sample rate/Mode1 calibrator rate) value of $\geqslant 1 \cdot 5$. Verification of a reaction was performed by retesting the filtrates, stored at $4-8^{\circ} \mathrm{C}$, within 48 hours of initial processing, using the IMx Select Chlamydia blocking reagent. A $50 \%$ or greater reduction in $\mathbf{S} / \mathbf{N}$ of the blocked specimen, compared with the $S / N$ of the reactive unblocked specimen, verified the presence of chlamydial antigen. The IMx processing time ranged from 45-70 minutes, depending on the number of samples tested.

\section{DISCREPANT SPECIMEN ANALYSIS}

Discrepant results between cell culture and the IMx Chlamydia assay were further investigated by performing the Syva Microtrak DFA test (Syva, Palo Alto, CA, USA), on the original MEIA specimen tube. Briefly, $1 \mathrm{ml}$ of $0.01 \mathrm{M}$ phosphate buffered saline (PBS), $\mathrm{pH}$ $7 \cdot 2$, was added to these samples. After vortexing, the swab was removed and the tube was 
Table 1 IMx Chlamydia versus Cell Culture as gold standard ${ }^{\star}$ and IMx Chlamydia and cell culture compared with a modified gold standard ¥

\begin{tabular}{|c|c|c|c|c|c|}
\hline Assay & Sensitivity & Specificity & $P P V$ & $N P V$ & Accuracy \\
\hline $\begin{array}{l}\text { IMx Chlamydia } \\
\text { (versus cell culture } \\
\text { as gold standard) }\end{array}$ & $\begin{array}{l}82 \cdot 1 \% \\
(23 / 28)\end{array}$ & $\begin{array}{l}99 \cdot 3 \% \\
(590 / 594)\end{array}$ & $\begin{array}{l}85 \% \\
(23 / 27)\end{array}$ & $\begin{array}{l}99 \cdot 2 \% \\
(590 / 595)\end{array}$ & $\begin{array}{l}98 \cdot 6 \% \\
(613 / 622)\end{array}$ \\
\hline $\begin{array}{l}\text { IMx Chlamydia } \\
\text { (compared with a } \\
\text { modified gold standard) }\end{array}$ & $\begin{array}{l}84 \cdot 4 \% \\
(27 / 32)\end{array}$ & $\begin{array}{l}100 \% \\
(590 / 590)\end{array}$ & $\begin{array}{l}100 \% \\
(27 / 27)\end{array}$ & $\begin{array}{l}99 \cdot 2 \% \\
(590 / 595)\end{array}$ & $\begin{array}{l}99 \cdot 2 \% \\
(617 / 622)\end{array}$ \\
\hline $\begin{array}{l}\text { Cell culture } \\
\text { (compared with a } \\
\text { modified gold standard) }\end{array}$ & $\begin{array}{l}87 \cdot 5 \% \\
(28 / 32)\end{array}$ & $\begin{array}{l}100 \% \\
(590 / 590)\end{array}$ & $\begin{array}{l}100 \% \\
(28 / 28)\end{array}$ & $\begin{array}{l}99 \cdot 4 \% \\
(590 / 594)\end{array}$ & $\begin{array}{l}99 \cdot 5 \% \\
(618 / 622)\end{array}$ \\
\hline
\end{tabular}

PPV = positive predictive value; NPV = negative predictive value.

*The prevalence rate by cell culture $=4 \cdot 5 \%(28 / 622)$.

†The modified gold standard was defined as a cell culture positive or an IMx Chlamydia positive confirmed with DFA and blocking reagent.

$\ddagger$ The prevalence rate by modified gold standard $=5 \cdot 1 \%(32 / 622)$.

then centrifuged at $5000 \times g$ for 30 minutes. A $30 \mu \mathrm{l}$ sample of the vortexed deposit was spotted onto an $8 \mathrm{~mm}$ slide well and processed according to the manufacturer's instructions. The presence of two or more elementary bodies was considered a positive confirmed result.

\section{STATISTICAL ANALYSIS}

Calculations of sensitivity, specificity, predictive values, and accuracy to determine test performance were based on standard methods. ${ }^{8}$ The statistical significance was calculated by the Fisher exact test.

\section{Results}

Endocervical swabs from 622 patients were assayed by the cell culture and the IMx Chlamydia. Table 1 shows the combined results of sensitivity, specificity, positive predictive value (PPV), negative predictive value (NPV), and accuracy, comparing the IMx Chlamydia against the cell culture as the gold standard and comparing the IMx Chlamydia and the cell culture with a modified gold standard. There were 27 samples positive by the IMx Chlamydia. In addition to these, a further two samples were reactive on initial testing, having $\mathrm{S} / \mathrm{N}$ values of 1.5 and $1 \cdot 6$, but did not verify positive, as the blocking result was less than $50 \%$. DFA testing was not performed on these two samples. The prevalence rate by cell culture was $4.5 \%(28 / 622)$.

Comparison of both methods with a modified gold standard was made. This was defined as a endocervical cell culture positive or endocervical IMx Chlamydia positive confirmed with the DFA test and the blocking reagent. There were 32 true positives out of the total 622 patient samples, 28 of which were detected by the cell culture method and 27 by

Table 2 Discrepant analysis

\begin{tabular}{lllll}
\hline Sample & $\begin{array}{l}\text { Cervical } \\
\text { cell culture }\end{array}$ & $\begin{array}{l}\text { IMx } \\
\text { Chlamydiat }\end{array}$ & $\begin{array}{l}\text { DFA on } \\
\text { MELA swab }\end{array}$ & $\begin{array}{l}\text { Urethral cell } \\
\text { culture }\end{array}$ \\
\hline 1 & $+++(62)$ & $-(1 \cdot 0)$ & - & $+++(65)$ \\
2 & $++(31)$ & $-(1 \cdot 1)$ & - & $+++(81)$ \\
3 & $++(17)$ & $-(1 \cdot 1)$ & - & $++(19)$ \\
4 & $++(10)$ & $-(1 \cdot 3)$ & - & - \\
5 & $+(4)$ & $-(1 \cdot 1)$ & + & $+++(42)$ \\
6 & - & $+(1 \cdot 7)$ & + & - \\
7 & - & $+(1 \cdot 7)$ & + & $+++(53)$ \\
8 & - & $+(2 \cdot 5)$ & + & \\
9 & + & $+(1 \cdot 6)$ & + \\
\hline
\end{tabular}

$\star$ Number of inclusion forming units per coverslip in parenthesis.

†MEIA S/ $\mathrm{N}$ value in parenthesis. the IMx Chlamydia method. The cell culture sensitivity was not significantly different from the IMx Chlamydia sensitivity $(p=0.998)$. The prevalence rate by the modified gold standard was $5 \cdot 1 \%(32 / 622)$.

A discrepancy analysis was performed on the nine samples that were positive by one method and negative by the other and the results are outlined in table 2 . The five samples that were culture positive IMx Chlamydia negative showed no elementary bodies in the MEIA tube when the DFA test was performed. The four culture negative IMx Chlamydia positive samples all showed elementary bodies in the MEIA transport tube when confirmed by the DFA test.

Also listed are the female urethral cell culture results from the discrepant samples. Of the 32 true positive endocervical samples, $59 \%(19 / 32)$ were positive by cell culture from the urethral site. A further nine patients were positive only from the urethral site, giving an overall prevalence rate of $6.6 \%(41 / 622)$. One patient was culture negative IMx Chlamydia positive and had been on antibiotics 4 days before examination. The nature of this antibiotic is unknown, but the patient had no previous history of chlamydia and no new contacts.

\section{Discussion}

The prevalence of chlamydia in Auckland has fallen dramatically from 1982 to 1993 , as seen in some other centres overseas. ${ }^{10}$ Changes in STD education programmes as part of HIV prevention strategies and local awareness of the morbidity associated with chlamydial infections, has led to increased numbers of patients attending for "check ups". From the mid 1980s, laboratory chlamydia screening tests have been widely available throughout New Zealand. Education campaigns through sexual health centres and family planning agencies has ensured increased diagnosis and appropriate therapy to the high risk youth group in particular. Recently, these services are seeing more asymptomatic patients of the informed low risk activity groups with a low prevalence of chlamydia. The reduction of chlamydial infection in New Zealand is paralleled by a fall in hospital admissions for pelvic inflammatory disease which reached its peak in 1986. ${ }^{11}$ There is a need for a continuing appraisal of non-culture chlamydial screening tests to monitor the effectiveness of chlamydia detection in our low prevalence populations.

Enzyme immunoassays, introduced for routine screening in the mid 1980s, have been widely used and developed to improve the detection of chlamydial infections. There have been numerous studies of enzyme immunoassays with reported ranges of sensitivities $62-98 \%$ and specificities $92-100 \%{ }^{12}$ The Abbott IMx Chlamydia offers an exciting new approach to chlamydia testing by using an automated IMx analyser, incorporating a unique microparticle capture detection system.

The cell culture method has traditionally been considered the gold standard against 
which other non-culture methods are compared. This highly specialised technique requires a high level of skill to maintain competency. Problems with this, combined with variabilities in methodology and stringent specimen requirements, mean that the gold standard is somewhat tarnished. The sensitivity of the cell culture method has been estimated to be no more than $70-80 \%$ for women with endocervical infection. ${ }^{13}$ Some now consider the gold standard aspect of the cell culture method to be a myth. ${ }^{12}$ It has now become more acceptable to compare methodologies against a group of tests as conducted by us and others. ${ }^{14-17}$ These have been called by a variety of names including modified gold standard, expanded gold standard, or infection status. This broadened gold standard approach has led to a greater recognition of the reliability of non-culture tests to detect disease, to an extent that non-culture tests are strongly underestimated if culture is used as the only reference. This is illustrated by our PPV results being $85 \cdot 2 \%$ if compared with culture only, or $100 \%$ if compared with the modified gold standard (table 1). Similar variations in PPVs has been shown by Thejls et al. ${ }^{17}$

As no one method is $100 \%$ sensitive, it is possible to detect more positives by using multiple assays than a single assay, as we and others have shown. ${ }^{16}$ This means there will be discrepancies between enzyme immunoassays and culture methods for a variety of reasons, including the quality and order of sampling, the effects of multiple sampling, storage conditions of specimens, and enzyme immunoassay/tissue culture sensitivity.

Also, the stage of the infection may have an influence on the test result. It is presumed that acute infection, with corresponding symptoms, is usually associated with high numbers of organisms, which should give a positive test result using most diagnostic tests. However, as the majority of women are asymptomatic for chlamydia, it is difficult to determine the association between the numbers of organisms present and the infection stage. Thomas $e t a l^{18}$ reported up to one third of clinical samples contain small numbers of chlamydia organisms. In the nine discrepant samples in our study, the majority would fall into the category of low numbers of organisms present, as determined by the number of inclusion forming units or Abbott IMx Chlamydia $\mathbf{S} / \mathrm{N}$ result. It seems likely that low level chlamydial infections are more difficult to identify by enzyme immunoassay, since our results in this study and others have shown that the performance of enzyme immunoassays is directly related to the number of inclusions found in culture. ${ }^{15} 1920$

Multiple site sampling to include the urethra is another very important aspect of chlamydia diagnosis. It is now recognised that performing chlamydia investigation from only one site will significantly underestimate the prevalence of chlamydia genital infection..$^{21} 22$ Our study supports this by finding an additional nine patients being diagnosed for chlamydia from a urethral cell culture specimen only. All of the endocervical samples in these nine cases were negative. The addition of a urethral sample in our study increased the number of positive patients having chlamydia by $28 \%(9 / 32)$. Jones et $a l^{23}$ has also reported similar findings where the addition of a urethral sample increased the prevalence of chlamydia by up to $25 \%$. Hay et $a^{21}$ also looked at both the endocervical and urethral site and concluded that both sites needed to be tested to define whether a woman was chlamydia positive. Other investigators, Sellors et al ${ }^{24}$ in a Canadian population, found only a $4.5 \%$ increase in the number of patients who were infected with chlamydia from the urethra. This suggests the importance of studying your own population group to determine the significance of the urethra as being the only site infected with chlamydia. The addition of a urethral swab with enzyme immunoassay screening in women should also show a similar increase in the prevalence rate, but this would require further investigation. Unpublished data in 1985, from chlamydia investigations in female patients attending the Auckland sexual health clinics, showed a prevalence of only $10 \%$ that were positive from the urethral site only. At that time it was suggested that all pregnant women, being screened before and after delivery, should have urethral cultures taken because there could be technical difficulties in acquiring an optimal endocervical specimen. It has also been suggested that as the prevalence of chlamydial infection decreases the urethral carriage increases, but this also would require further investigation.

The performance of the Abbott IMx Chlamydia assay, as shown by our results, was excellent. Other studies evaluating the assay have shown similar high levels of performance. ${ }^{25} 26$ In particular, a large multicentre study by Baselski et al, showed the sensitivity, specificity, PPV, and NPV to be $91.8 \%$, $99 \cdot 1 \%, 94.0 \%$, and $98 \cdot 8 \%$, respectively. The discrepant analysis in this study was performed by DFA or polymerase chain reaction.

Confirmation testing of all EIA reactive specimens is important. This is highlighted in our study where two samples, although only slightly above the cut off, did not verify positive after blocking. The number of samples this represented was $7 \%(2 / 29)$ of the initial reactives, which is significant and consistent with the recommendation from the manufacture to confirm reactives.

During the discrepant specimen analysis in our study, where the original MEIA specimen tube was used, it was decided not to perform the DFA test on the MEIA filtrates that were culture positive IMx Chlamydia negative, at the recommendation of the manufacturer. This is because the filtration process may have the undesirable effect of trapping elementary bodies (personal communication with Abbott Laboratories during study). Further investigation would be required to determine whether or not there would be any loss of detectable particles during filtration.

The Abbott IMx system offers a large range of diagnostic tests. The IMx Chlamydia method was relatively straightforward but 
included a special filtration step during the processing of the sample before loading the filtrate into the analyser. The batch size of 24 samples per run consisted of 22 patients and two controls. This would be limiting for laboratories with large numbers of specimens to process (100-200 per day), but could be overcome to a degree by the installation of two or more analysers. The instrument would be best suited to small to medium size laboratories where the number and flexibility of the tests would cater for their requirements.

The sensitivity, specificity, PPV, and accuracy of results of our study show that the Abbott IMx Chlamydia assay is an excellent method for diagnosing chlamydial infection. As there are many variables that can affect the final chlamydial result, it is essential to control every aspect in the procedure from initial collection to confirmation of a reactive result.

We would like to thank Dr David Bremner and Dr Murray Reid for their valuable contribution to this paper. We are also grateful to Abbott for provision of equipment and materials.

1 Say PJ, Hookham AB, Willmott FE. Unsuspected Chlamydia trachomatis in females attending a sexually Chlamydia trachomatis in females attending a sexuall

2 Centres for Disease Control. Recommendations for the prevention and management of Chlamydia trachomatis infections. $M M W R$ 1993;42:RR-12.

3 Ridgway GL, Taylor-Robinson D. Current problems in microbiology: I Chlamydial infections: Which laboratory test ? F Clin Pathol 1991;44:1-5.

4 Schwebke JR, Stamm WE, Handsfield HH. Use of sequential enzyme immunoassay and direct fluorescent antibody tests for detection of Chlamydia trachomatis infection in women. $\mathcal{F}$ Clin Microbiol 1990;28:2473-6.

5 Stamm WE, Tam M, Koester M, Cles L. Detection of Chlamydia trachomatis inclusions in McCoy cell cultures with fluorescein-conjugated monoclonal antibodies. with Hiorescein-conjugated

6 Mårdh P-A, Weström L, Colleen S, Wølner-Hanssen P. Sampling, specimen handling and isolation techniques in the diagnosis of chlamydia and other genital infections. Sex Transm Dis 1981;8:280-5.

7 Fiore M, Mitchell J, Doan T, Nelson R, Winter G Grandone $C$, et al. The Abbott IMx ${ }^{T M}$ automated benchtop immunochemistry analyser system. Clin Chem 1988; 34:1726-32.

8 Department of Clinical Epidemiology and Biostatistics, McMaster University Health Science Centre, Hamilton, Ontario: How to read clinical journals: II. To learn about a diagnostic test. Can Med Assoc f 1981;124:703-10.

9 Larsson P-G, Platz-Christensen J-J, Thejls H, Forsum U, Påhlson C. Incidence of PID following first trimester legal abortion in women with bacterial vaginosis after treatment with metronidazole; a double-blind randomised study. Am ๆ Obstet Gynecol 1992;166:100-3

10 Thejls H, Gnarpe J, Gnarpe H, Larsson G. Age-related decrease in prevalence of Chlamydia trachomatis among pregnant women. Sex Transm Dis 1991;18:137.

11 Farquhar C, Jamieson M, eds. In: Introduction to obstetric and gynaecology, 1st ed. Auckland: Department of Obstetrics and Gynaecology, University of Auckland 1994:175-9.

12 Taylor-Robinson D, Thomas BJ. Laboratory techniques for the diagnosis of chlamydial infection. Genitourin Med 1991;67:256-66.

13 Schachter J. Biology of Chamydia trachomatis. In: Holmes $\mathrm{KK}$, Mårdh P-A, Sparling PF, Wiesner PJ, eds. Sexually transmitted diseases. 2nd ed. New York: McGraw Hill, transmitted diseas

14 Noble MA, Kwong A, Barteluk RL, Smith RP. Laboratory

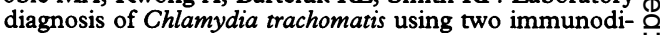
agnostic methods. Am $\mathcal{F}$ Clin Pathol 1988;90:205-10.

15 Magder LS, Klontz KC, Bush LH, Barnes RC. Effect of $\overrightarrow{\vec{\overrightarrow{ }}}$ patient characteristics on performance of an enzyme mmunoassay for detecting cervical Chlamydial trachomatis infection. 7 Clin Microbiol 1990;28:781-4.

16 Kluytmans JAJW, van der Willigen AH, van Heyst BYM, $\overline{\bar{C}}$ van der Meyden WI, Stolz E, Wagenvoort JH, et al. Evaluation of an enzyme immunoassay for detection of $\mathbb{D}$ Chlamydia trachomatis in urogenital specimens. Int $¥$ STD AIDS 1990;1:49-52.

17 Thejls H, Gnarpe J, Gnarpe H, Larsson PG, PlatzChristensen JJ, Östergaard L, et al. Expanded gold stan- . dard in the diagnosis of Chlamydia trachomatis in a low prevalence population: diagnostic efficacy of tissue cul- $\vec{\omega}$ ture, direct immunofluorescence, enzyme immunoassay, PCR and serology. Genitourin Med 1994;70:300-3

18 Thomas BJ, Osborne MF, Gilchrist C, Taylor-Robinson D. Improved sensitivity of an enzyme immunoassay IDEIA for detecting Chlamydia trachomatis. $\mathcal{F}$ Clin Pathol 1989;42:759-62.

19 Amortegui AJ, Meyer MP. Enzyme immunoassay for $\overrightarrow{0}$ detection of Chlamydia trachomatis from the cervix. Obstet $\infty$ Gynaecol 1985;65:523-6.

20 Smith JW, Rogers RE, Katz BP, Brickler JF, Lineback PI Van der Pol B, et al. Diagnosis of chlamydial infection in women attending antenatal and gynaecologic clinics. $f$. women attending antenatal and
Clin Microbiol 1987;25:868-72.

21 Hay PE, Thomas BJ, Horner PJ, MacLeod E, Renton AM, Taylor-Robinson D. Chlamydia trachomatis in women: the more you look, the more you find. Genitourin Med $\Phi$ 1994;70:97-100.

22 Ridgway GL, Mumtaz G, Robinson AJ, Franchini $M, \vec{C}$ Carder C, Burczak J, Lee H. Comparison of the ligase chain reaction with cell culture for the diagnosis of. Chlamydia trachomatis infection in women. $\mathcal{F}$ Clin Pathol 1996;49:116-9.

23 Jones RB, Katz BP, van der Pol B, Caine VA, Batteiger BE, Newhall WJ. Effect of blind passage and multiple samNewhall WJ. Effect of blind passage and multiple sampling on recovery of Chlamydia trachomatis from

24 Sellors JW, Mahony JB, Jang D, Pickard L, Goldsmith $\mathrm{CH}$, Gafni $A$, et al. Comparison of cervical, urethral and urine specimens for the detection of Chlamydia trachomatis in women. F Infect Dis 1991;164:205-8.

25 Baselski V, Jungkind D, Chernesky M, Sedmark G, Bodner J. Multi-centre evaluation of the Abbott IMx Select ${ }^{\mathbb{8}}$ Chlamydia assay for detection of Chlamydia trachomatis in female endocervical and male urethral swabs. of Klinisches Labor 1995;41:373-7.

26 Sedmak G, Koshakow M, Abbott G, Bodner J, Brotherton $\delta$ $\mathrm{D}$, Kolczaski $\mathrm{M}$, et al. Two comparative studies of the Abbott IMx Select ${ }^{\mathbb{B}}$ Chlamydia assay with endocervical Abbott IMx Select ${ }^{\mathbb{B}}$ Chlamydia assay with endocervical $\delta$ specimens, one versus the SYVA Microtrak II Chlamydia
EIA assay and another versus the VIDAS Chlamydia EIA assay and another versus the VIDAS Chlamydia
assay. Klinisches Labor 1995;41:379-81. 